\title{
A Prospective Observational Study in a Tertiary Care Hospital to Evaluate the Utility of Pleural Fluid Cholesterol and Lactate Dehydrogenase (LDH) in Differentiating Exudative from Transudative Pleural Effusions
}

\section{Sujith Halappa1, Gayathri Devi HJ2*, Majeed Pasha MD $^{3}$ and Padmaja $\mathrm{MS}^{4}$}

${ }^{1}$ Assistant Professor, Department of Respiratory Medicine, Subbaiah Institute of Medical Sciences, Shivamogga, Karnataka, India

${ }^{2}$ Professor and Head, Department of Respiratory Medicine, M.S. Ramaiah Medical College, Bengaluru, Karnataka, India ${ }^{3}$ Assistant Professor, Department of Respiratory Medicine, Raja Rajeshwari Medical College, Bengaluru, Karnataka, India ${ }^{4}$ Senior Resident (D.M), Department of Pulmonary Medicine and Critical Care, AIIMS Bhubaneswar, India

*Corresponding author: Gayathri Devi HJ, Professor and Head, Department of Respiratory Medicine, M.S. Ramaiah Medical College, Bengaluru, Karnataka, India, Tel: +919916195235; Email: gayathrijoshy@gmail.com

\section{Abstract}

Background: Light's criteria have been the gold standard to differentiate exudative from transudative pleural effusions. It requires biochemical estimations from both pleural fluid and serum which may not be feasible in all patients due to economic constraints. Pleural fluid cholesterol and Lactate Dehydrogenase (LDH) have been reported to be extremely useful in distinguishing between transudative and exudative pleural effusion.

Methods: This was a Prospective Observational study carried out at a tertiary care hospital. In this study with a sample size of 76, pleural fluid cholesterol and LDH were analysed in patients with pleural effusion. Etiological diagnosis was based on clinical history with radiological imaging, biochemical and cytological examination of pleural fluid.

Results: The study included 76 patients with $69.7 \%(n=53)$ males and $30.3 \%(n=23)$ females. The mean age of patients was $48.97+17.03$ years. Of 76 cases, 62 were exudates and 14 transudates. The sensitivity and specificity of pleural fluid cholesterol ( $>45 \mathrm{mg} / \mathrm{dl}$ ) for distinguishing exudates from transudates was 98.4\% and $86 \%$, while for LDH ( $>200 \mathrm{mg} / \mathrm{dl})$, 93.5\% and 78.6\% respectively. Pleural Fluid cholesterol and LDH together (Costa's Criteria) had a sensitivity of 95\% and specificity of $93 \%$ for differentiating effusions in comparison to gold standard Light's criteria which had sensitivity and specificity of $100 \%$ and $71.4 \%$ which was statistically significant. 


\section{Open Access Journal of Pulmonary \& Respiratory Sciences}

Conclusion: Pleural fluid cholesterol and LDH were extremely sensitive and specific as well as highly cost effective for differentiating exudative from transudative effusions.

Keywords: Cholesterol; Lactate dehydrogenase; Light's criteria; Costa's criteria

Abbreviations: LDH: Lactate Dehydrogenase; ADA: Adenosine Deaminase; ANOVA: Analysis of Variance; PPV: Positive Predictive Value; NVP: Negative Predictive Value.

\section{Introduction}

Pleural Effusion is a commonly encountered clinical problem by Pulmonologists as well as physicians. The management strategy to be adopted in pleural effusion depends on whether an effusion is a transudate or exudate. It is very important to establish accurate etiological diagnosis to treat the patient in an appropriate manner as about 15 to $20 \%$ of cases remain undiagnosed [1].

Though there are many criteria to classify pleural effusions as transudative or exudative, the most commonly and extensively used has been Light's criteria. The validity and reliability of other criteria for example the one to be used in the present study (Pleural Fluid Cholesterol and LDH) requires evaluation. There are many studies in Western literature but very few studies in Indian literature, which have assessed the role of pleural fluid cholesterol $(>45 \mathrm{mg} / \mathrm{dl})$ and lactate dehydrogenase $(>200 \mathrm{mg} / \mathrm{dl}$ ) in differentiating between transudative and exudative pleural effusions. Using only cholesterol and lactate dehydrogenase level lowers the cost of the diagnostic procedure which is important in developing countries like India. Hence there is a role for defining the best cost effective and diagnostic approach for quicker diagnosis of pleural effusion and the need for the present study.

The pleural fluid LDH is a reliable indicator of degree of pleural fluid inflammation. Higher the pleural fluid $\mathrm{LDH}$, the more inflamed the pleural surfaces [2]. Serial measurements of pleural fluid LDH is useful in undiagnosed pleural effusions. It is also useful in differentiating exudates and transudates [2]. Pleural fluid cholesterol has been reported to be useful in distinguishing between transudative and exudative pleural effusion. Various causes may be responsible for the presence of cholesterol in the pleural effusion [3-5]. However, till recently, the cholesterol content of pleural fluid had been used along with the concentration of other lipid fractions to distinguish between chylothorax and pseudochylothorax.. Few studies have shown that pleural fluid cholesterol levels differ significantly in transudative and exudative pleural effusion [6-10]. These studies have shown a high sensitivity and specificity for pleural fluid cholesterol levels to differentiate between transudative and exudative effusion.

We aimed to use the criteria of pleural fluid cholesterol and LDH levels to distinguish between transudative and exudative pleural effusion.

\section{Aim of the Study}

To compare Costa's Criteria (Pleural Fluid Cholesterol $>45 \mathrm{mg} / \mathrm{dl}$ and LDH $>200 \mathrm{mg} / \mathrm{dl}$ ) with Light's criteria in differentiating exudative from transudative pleural effusions.

\section{Objectives of the Study}

1. To determine pleural fluid Cholesterol and LDH from pleural fluid analysis.

2. To determine the cut-off levels for pleural fluid cholesterol and LDH in our set up, given the variability of results from one laboratory to another.

\section{Study Methods}

1. 76 patients with Pleural Effusion were included over a period of 18 months from January 2015 to June 2016 at a tertiary care hospital.

2. Demographic data, detailed clinical history, and clinical examination findings were recorded.

3. Relevant laboratory tests including Chest radiograph, ultrasonography, Serum Cholesterol and LDH were performed in all patients.

4. Pleural Fluid Analysis included levels of protein, sugar, adenosine deaminase (ADA), cholesterol, LDH with cytological and microbiological examination.

5. Additional investigations such as computed tomography chest, pleural biopsy, bronchoscopy, 2-D echocardiogram were done wherever indicated. 


\section{Open Access Journal of Pulmonary \& Respiratory Sciences}

\section{Study Subjects}

\section{Inclusion Criteria}

1. Patients aged $>18$ years with pleural effusion.

2. Patients diagnosed with Pleural Effusion based on history, clinical examination and appropriate imaging modality (Chest radiography, USG Chest, CT Scan Chest).

\section{Exclusion Criteria}

1. Patients who were hemodynamically unstable.

2. Previous Thoracentesis

3. Traumatic pleural tap

4. Procedure related pleural effusion

5. Suspected Pulmonary Thromboembolism

6. Chylothorax, Haemothorax

7. Patients who are unwilling to participate in the study

\section{Statistical Analysis}

\section{Statistical Methods}

Descriptive and inferential statistical analysis has been carried out in the present study. Results on continuous measurements are presented on Mean + SD (Min-Max) and results on categorical measurements are presented in Number (\%). Significance is assessed at $5 \%$ level of significance. The following assumptions on data are made, Assumptions: 1 . Dependent variables should be normally distributed, 2. Samples drawn from the population should be random, and Cases of the samples should be independent.

Analysis of variance (ANOVA) has been used to find the significance of study parameters between three or more groups of patients. Student $t$ test (two tailed, independent) has been used to find the significance of study parameters on continuous scale between two groups (Inter group analysis) on metric parameters.

Chi-square/ Fisher Exact test has been used to find the significance of study parameters on categorical scale between two or more groups.

Significant figures

+ Suggestive significance ( $\mathrm{p}$ value: $0.05<\mathrm{p}<0.10$ )

* Moderately significant ( $p$ value: $0.01<p<0.05$ )

$* *$ Strongly significant ( $p$ value: $p<0.01$ )

\section{Statistical Software}

The Statistical software namely SAS 9.2, SPSS 15.0, Stata 10.1, MedCalc 9.0.1, Systat 12.0 were used for the analysis of the data and Microsoft word and Excel have been used to generate graphs, Tables etc .

\section{Results}

Of 76 patients evaluated, 53 were males and 23 females (Table 1). Mean age among patients was $48.97+17.03$ yrs. Of 76 cases of pleural effusion, $62(82 \%)$ were exudative and 14(18\%) transudative (Table 2). Tuberculosis was the most common cause of exudative effusion and Chronic Kidney Disease among transudative effusions (Tables 3 and 4). Mean pleural fluid glucose in exudative effusion was 107.92 and in transudative pleural effusion was $116.57+69.57$, while mean pleural fluid protein was $4.24+1.41$ and $1.84+0.66$ in exudative and transudative effusions (Tables 5 and 6). The Cholesterol in exudative pleural fluid was $81.36+5.30$ and in transudative pleural fluid $26.25+3.64$ (Tables 7 and 8 ) which was strongly significant $(\mathrm{p}<0.001)$. Mean LDH in exudative and transudative effusions was $772.52+144.56$ and $116.05+32.89$ respectively (Tables 7 and 8 ) which was moderately significant $(\mathrm{p}<0.035)$.

Pleural fluid cholesterol $(>45 \mathrm{mg} / \mathrm{dl})$ had a sensitivity, specificity, PPV, NPV of 98\%, 86\%, 97\%, 92\% in differentiating exudates while $\mathrm{LDH}(>200 \mathrm{mg} / \mathrm{dl})$ had a sensitivity, specificity, PPV, NPV of $93.5 \%, 77 \%, 95 \%$ and $73 \%$ (Table 9) which were strongly significant statistically $(\mathrm{p}<0.001)$. The Pleural Fluid LDH and Cholesterol (Costa's criteria) had a sensitivity, specificity, PPV, NPV of $95 \%, 93 \%, 98 \%, 81 \%$ compared to gold standard Light's criteria which had a sensitivity, specificity, PPV, NPV of $100 \%, 71 \%, 94 \%$ and $100 \%$ (Table 10) being strongly significant statistically. In addition pleural fluid/serum Cholesterol ratio $(>0.4)$ had a sensitivity, specificity, PPV, NPV of 74\%, 79\%, 94\%, 40\% in differentiating exudates (Table 11). Using ROC curve analysis, cut off levels were drawn from the study for pleural fluid cholesterol at $36 \mathrm{mg} \%$ and pleural fluid LDH at $140 \mathrm{mg} \%$ which was highly sensitive and specific and with AUC $>90 \%$ appears to be an excellent test in differentiating exudative and transudative effusions(Tables 12 and 13).

\begin{tabular}{|c|c|c|}
\hline Gender & No. of patients $(\mathbf{n}=\mathbf{7 6})$ & $\mathbf{\%}$ \\
\hline Male & 53 & 69.7 \\
\hline Female & 23 & 30.3 \\
\hline
\end{tabular}

Table 1: Gender distribution of patients. 


\section{Open Access Journal of Pulmonary \& Respiratory Sciences}

\begin{tabular}{|c|c|c|}
\hline Type of Pleural effusion & No. of patients (n=76) & $\mathbf{\%}$ \\
\hline Exudate & 62 & 82 \\
\hline Transudate & 14 & 18 \\
\hline
\end{tabular}

Table 2: Type of Pleural effusion.

\begin{tabular}{|c|c|c|}
\hline & No. of patients & $\mathbf{\%}$ \\
\hline Tubercular & 39 & 51.3 \\
\hline Parapneumonic & 13 & 17.1 \\
\hline Paramalignant & 5 & 6.6 \\
\hline Malignant & 3 & 3.9 \\
\hline Undiagnosed & 2 & 2.6 \\
\hline
\end{tabular}

Table 3: Type of Exudative pleural effusion.

(NOTE: The term Paramalignant effusion refers to effusions which are not the result of direct neoplastic involvement of pleura but are still related to the primary tumour)

\begin{tabular}{|c|c|c|}
\hline & No. of patients $(\mathbf{n}=\mathbf{7 6})$ & $\mathbf{\%}$ \\
\hline CKD & 10 & 13.15 \\
\hline CLD & 2 & 2.6 \\
\hline CCF & 2 & 2.6 \\
\hline
\end{tabular}

Table 4: Type of Transudative pleural effusion.

\begin{tabular}{|c|c|}
\hline Variables & Mean + SD \\
\hline PF Glucose & 107.92 \\
\hline PF protein & $4.24+1.41$ \\
\hline
\end{tabular}

Table 5: Analysis of pleural fluid parameters in Exudative pleural effusions.

ANOVA test, Mean \pm SD

\begin{tabular}{|c|c|}
\hline Variables & Transudate \\
\hline PF Glucose & $116.57 \pm 69.57$ \\
\hline PF protein & $1.84 \pm 0.66$ \\
\hline
\end{tabular}

Table 6: Analysis of pleural fluid parameters in Transudative pleural effusions.

\begin{tabular}{|c|c|c|c|c|}
\hline \multirow{2}{*}{ Variables } & \multicolumn{2}{|c|}{ Exudate } & \multirow{2}{*}{ Total } & \multirow{2}{*}{ P value } \\
\cline { 2 - 5 } & Yes & No & $651.59 \pm 121.51$ & $0.035^{*}$ \\
\hline PF LDH & $772.52 \pm 144.56$ & $116.05 \pm 32.89$ & $71.22 \pm 5.01$ & $<0.001^{* *}$ \\
\hline PF Cholesterol & $81.36 \pm 5.30$ & $26.25 \pm 3.64$ &
\end{tabular}

Table 7: Analysis of PF LDH and PF Cholesterol in Exudative effusions.

Mean \pm SE, student $t$ test

\begin{tabular}{|c|c|c|c|c|}
\hline \multirow{2}{*}{ Variables } & \multicolumn{2}{|c|}{ Transudate } & \multirow{2}{*}{ Total } & \multirow{2}{*}{ P value } \\
\cline { 2 - 3 } & Yes & No & $551.59 \pm 144.56$ & $0.035^{*}$ \\
\hline PF LDH & $116.06 \pm 32.89$ & $772.52 \pm 144.56$ & $71.22 \pm 5.01$ & $<0.001^{* *}$ \\
\hline PF Cholesterol & $26.25 \pm 3.64$ & $81.36 \pm 5.30$ &
\end{tabular}

Table 8: Analysis of PF LDH and PF Cholesterol in Transudative effusions.

Mean \pm SE Student $t$ test

\begin{tabular}{|c|c|c|c|c|c|c|c|c|c|c|c|}
\hline \multirow{2}{*}{ Variables } & \multicolumn{4}{|c|}{ Observation } & \multicolumn{4}{|c|}{ Correlation (in \%) } \\
\cline { 2 - 12 } & TP & FP & FN & TN & Total & Se & S p & PPV & NPV & Accuracy & P value \\
\hline Pleural fluid cholesterol & 61 & 2 & 1 & 12 & 76 & 98 & 86 & 97 & 92 & 96 & $<0.001^{* *}$ \\
\hline Pleural fluid LDH & 58 & 3 & 4 & 11 & 76 & 94 & 79 & 95 & 73 & 91 & $<0.001^{* *}$ \\
\hline
\end{tabular}

Table 9: Sensitivity (Se), Specificity (Sp), Positive Predictive Value (PPV), Negative Predictive Value(NPV) of Pleural fluid Cholesterol $(>45 \mathrm{mg} / \mathrm{dl})$ and $\mathrm{LDH}(>200 \mathrm{mg} / \mathrm{dl})$ in differentiating Exudates from Transudates.

(Note: TP-True positive, FP-False positive, FN-False negative, TN-True negative)

\begin{tabular}{|c|c|c|c|c|c|c|c|c|c|c|c|}
\hline \multirow{2}{*}{ Criteria } & \multicolumn{4}{|c|}{ Observation } & \multicolumn{6}{|c|}{ Correlation (in \%) } \\
\cline { 2 - 13 } & TP & FP & FN & TN & Total & Se & S p & PPV & NPV & Accuracy & P value \\
\hline Light's Criteria & 62 & 4 & 0 & 10 & 76 & 100 & 71 & 94 & 100 & 95 & $<0.001^{* *}$ \\
\hline Costa's Criteria & 59 & 1 & 3 & 13 & 76 & 95 & 93 & 98 & 81 & 95 & $<0.001^{* *}$ \\
\hline
\end{tabular}

Table 10: Sensitivity (Se), Specificity (Sp), Positive Predictive Value (PPV), Negative Predictive Value (NPV) of Light's Criteria and Costa's Criteria in differentiating Exudates from Transudates. 


\section{Open Access Journal of Pulmonary \& Respiratory Sciences}

\begin{tabular}{|c|c|c|c|c|c|c|c|c|c|c|}
\hline \multirow{2}{*}{ Criteria } & \multicolumn{4}{|c|}{ Observation } & \multicolumn{6}{|c|}{ Correlation (in \%) } \\
\hline & $\mathbf{T P}$ & FP & \begin{tabular}{|l|l|} 
FN & TN \\
\end{tabular} & Total & Se & Sp & PPV & NPV & Accuracy & P value \\
\hline Pleural fluid/Serum cholesterol ratio $(>0.4)$ & 46 & 3 & \begin{tabular}{|l|l|}
16 & 11 \\
\end{tabular} & 76 & 74 & 79 & 94 & 40 & 75 & $>0.05$ \\
\hline
\end{tabular}

Table 11: Sensitivity (Se), Specificity (Sp), Positive Predictive Value (PPV), Negative Predictive Value (NPV) of pleural fluid/serum cholesterol ratio for differentiating exudative from transudative pleural effusion.

\begin{tabular}{|c|c|c|c|c|c|c|c|c|}
\hline \multirow{2}{*}{ Variables } & \multicolumn{4}{|c|}{ ROC results to Exudate } & \multirow{2}{*}{ Cut-off } & \multirow{2}{*}{ AUROC } & \multirow{2}{*}{ SE } & \multirow{2}{*}{ P value } \\
\cline { 2 - 8 } & Sensitivity & Specificity & LR+ & LR- & & & \\
\hline PF LDH & 96.77 & 85.71 & 6.77 & 0.04 & $>140$ & 0.944 & 0.05 & $<0.001^{* *}$ \\
\hline PF Cholesterol & 96.77 & 92.86 & 13.6 & 0.04 & $>36$ & 0.969 & 0.03 & $<0.001^{* *}$ \\
\hline
\end{tabular}

Table 12: ROC curve analysis in Exudative Effusions.

\begin{tabular}{|c|c|c|c|c|c|c|c|c|}
\hline \multirow{2}{*}{ Variables } & \multicolumn{4}{|c|}{ ROC results to Transudate } & \multirow{2}{*}{ Cut-off } & \multirow{2}{*}{ AUROC } & \multirow{2}{*}{ SE } & \multirow{2}{*}{ P value } \\
\cline { 2 - 9 } & Sensitivity & Specificity & LR+ & LR- & & & & \\
\hline PF LDH & 92.86 & 96.77 & 26.6 & 0.2 & $\leq 140$ & 0.944 & 0.05 & $<0.001^{* *}$ \\
\hline PF Cholesterol & 92.96 & 96.77 & 28.8 & 0.1 & $\leq 36$ & 0.969 & 0.02 & $<0.001^{* *}$ \\
\hline
\end{tabular}

Table 13: ROC curve analysis in Transudative Effusions.

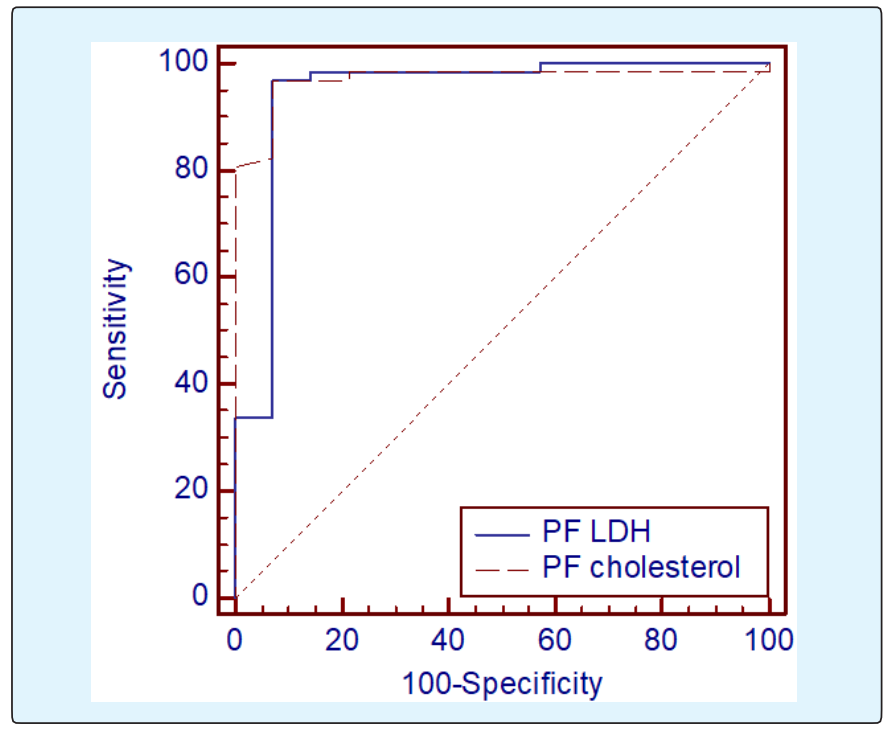

\section{Discussion}

A prospective observational study was conducted on pleural effusion patients in a tertiary care hospital. Pleural effusion is a commonly encountered clinical problem by Pulmonologists as well as physicians caused by diverse pathological conditions. It is very important to establish accurate etiological diagnosis to treat the patient in an appropriate manner as about 15 to $20 \%$ of cases remain undiagnosed [1]. A better knowledge of spectrum of clinical history, radiological imaging, biochemical and cytological evaluation helps in narrowing down diagnostic dilemma and aid in management of patients. Our aim was to evaluate the role of pleural fluid
Cholesterol and LDH in differentiating exudative and transudative effusions. There are many studies in western literature but very few Indian studies which have assessed the role of pleural fluid Cholesterol and LDH in differentiating between transudative and exudative pleural effusions. Results obtained from the study were compared with the studies in the literature.

\section{Pleural Fluid Cholesterol}

In our study mean cholesterol level in exudative pleural effusion was $81.36+5.30$ and in transudative pleural effusion was $26.35+3.64$ with sensitivity $98.4 \%$, specificity $86 \%$, PPV $96.8 \%$ and NPV $92 \%$ for distinguishing exudates and transudates. In a similar study by Rogerio, et al. [11] the mean fluid cholesterol level in the exudates was $90.39 \mathrm{mg} / \mathrm{dl}$ with sensitivity of $97.22 \%$, a specificity of $85.71 \%$, a positive predictive value of $98.59 \%$ and a negative predictive value of $75 \%$. A meta-analysis by Shen, et al. [12] found fluid cholesterol had a sensitivity $88 \%$ and specificity $96 \%$. In another study by Guleria, et al. [1] role of pleural fluid cholesterol was evaluated in 50 patients in differentiating transudates and exudates and found that pleural fluid cholesterol had a sensitivity of $88 \%$ and specificity of $100 \%$ for exudates with an accuracy of $92 \%$.

\section{Pleural Fluid LDH}

The mean pleural fluid LDH among exudative effusions was $772.52 \pm 144.56$, and in transudative effusions 116.06 +32.89 with sensitivity and specificity of $93.5 \%$ and $78.6 \%$ which was similar to study by Rungta and Jha [13] 


\section{Open Access Journal of Pulmonary \& Respiratory Sciences}

in which mean pleural fluid LDH in transudative effusions was $95+24.5$ with sensitivity of $79 \%$ and specificity $75 \%$ in differentiating exudative effusions. In contrast a study by Guleria, et al. [1] which included 50 patients, pleural fluid LDH had a sensitivity of $80 \%$ and specificity of $96 \%$ for exudates.

\section{Comparing Pleural Fluid LDH/Cholesterol (Costa's Criteria) and Light's Criteria}

Light's criteria have been the gold standard to differentiate exudative and transudative effusions. There are many criterias to classify pleural effusions as transudative or exudative like the one used in the present study such as Costa's criteria. From the present study Sensitivity, Specificity, Positive Predictive Value (PPV) and Negative Predictive Value of Costa's Criteria for distinguishing exudates and transudates was $97 \%, 86 \%$, $95.24 \%, 86 \%$ compared to sensitivity, specificity, PPV,NPV of Light's criteria which was 100\%, 71\% , 93.9\% and $100 \%$ with statistically significant $p$ values . So as is evident from the study Costa's criteria is almost equally sensitive and more specific as Light's criteria in differentiating exudates and transudates.

In a similar study by Judith and Jorge [14] who studied the role of pleural fluid cholesterol and lactate dehydrogenase (COSTA'S Criteria: pleural fluid cholesterol $>45 \mathrm{mg} / \mathrm{dl}, \mathrm{LDH}>200 \mathrm{IU} / \mathrm{L}$ ) in differentiating pleural effusion as transudate and exudates, found that sensitivity and specificity of COSTA'S Criteria when compared with gold standard (LIGHT'S Criteria) was 73\% and $100 \%$ respectively. This study comparatively showed higher specificity but lower sensitivity with respect to our study.

In another study by Rungta and Jha [13] who as well studied the diagnostic value of pleural fluid cholesterol and LDH in differentiating transudate and exudate concluded from their study that pleural fluid cholesterol and lactate dehydrogenase had a sensitivity of $99 \%$ and specificity of $98 \%$ in separating transudate and exudate. This study showed a similar sensitivity but higher specificity compared to our study.

\section{Pleural Fluid/Serum Cholesterol Ratio}

In our study pleural fluid/serum Cholesterol ratio $(>0.4)$ had a sensitivity, specificity, PPV, NPV of $74 \%$, $79 \%, 94 \%, 40 \%$ in differentiating exudates from transudates. In a similar study by Rogerio, et al. [11] which included 79 patients with exudative effusion, the PC/SC ratio demonstrated a sensitivity of $81.48 \%$, a specificity of $57.14 \%$, and a positive predictive value of $93.61 \%$ and a negative predictive value of $28.57 \%$. In contrast in a meta-analysis by Yougchun Shen, et al. [14] which included 20 studies $\mathrm{P} / \mathrm{S}$ cholesterol ratio had a sensitivity $94 \%$ and specificity $87 \%$.

\section{Conclusion}

As is evident from the literature and from the present study Costa's Criteria (Pleural fluid Cholesterol $>45 \mathrm{mg} / \mathrm{dl}$ and LDH $>200 \mathrm{mg} / \mathrm{dl}$ ) is equally sensitive and more specific in differentiating exudates and transudates compared to gold standard Light's Criteria. It is also more economical, reducing the burden of additional investigations as required in Light's criteria.

\section{References}

1. Guleria R, Agarwal SK, Sinha S, Pande JN, Mishra A (2003) Role of pleural fluid cholesterol in differentiating transudative from exudative pleural effusion. Nat Med J India 16(2): 64-69.

2. Light RW (2013) Clinical manifestations and useful tests. $6^{\text {th }}$ (Edn.), Pleural Diseases. Lippincott Williams and Wilkins, pp: 99-101.

3. Hamm H, Brohan U, Bohmer R, Missmahl HP (1987) Cholesterol in pleural effusions: A diagnostic aid. Chest 92(2): 296-302.

4. Valdes L, Pose A, Suarez J, Gonzalez-Juanatey JR, Sarandeses A, et al. (1991) Cholesterol: A useful parameter for distinguishing between pleural exudates and transudates. Chest 99(5): 1097-1102.

5. Stein HM (1932) Cholesterol thorax in tuberculosis (cholesterol pleurisy). Arch Intern Med 49(3): 421428.

6. Ferguson GC (1966) Cholesterol pleural effusion in rheumatoid lung disease. Thorax 21: 577-582.

7. Coe JE, Aikawa JK (1961) Cholesterol pleural effusion. Report of 2 cases studied with isotopic techniques and review of the world literature. Arch Intern Med 108: 763-774.

8. Vaz MA, Marchi E, Vargas FS (2001) Cholesterol in the separation of transudates and exudates. Curr Opin Pulm Med 7(4): 183-186. 


\section{Open Access Journal of Pulmonary \& Respiratory Sciences}

9. Jimenez Castro D, Diaz Nuevo G, Perez-Rodriguez E (2002) Comparative analysis of Light's criteria and other biochemical parameters to distinguish exudates from transudates. Rev Clin Esp 202(1): 3-6.

10. Gazquez I, Porcel JM, Vives M, Vicente de Vera MC, Rubio M, et al. (1998) Comparative analysis of Light's criteria and other biochemical parameters for distinguishing transudates from exudates. Respir Med 92(5): 762-765.

11. Rufino R, Marques BL, Azambuja Rde L, Mafort T, Pugliese JG, et al. (2014) Pleural Cholesterol to the Diagnosis of Exudative Effusion. Open Respir Med J 8: 14-17.
12. Shen Y, Zhu H, Wan C, Chen L, Wang T, et al. (2014) Can cholesterol be used to distinguish pleural exudates from transudates? evidence from a bivariate meta-analysis. BMC Pulm Med 14: 61.

13. Rohit Rungta, Rajendra K Jha (2013) Comparitive analysis of pleural fluid biochemical parameters with cholesterol to differentiate transudates from exudates. J Assoc Chest Physicians 1(2): 54-57.

14. Judith S Soliano, Manuel C Jorge (2008) Comparing pleural fluid cholesterol and lactate dehydrogenase (Costa's criteria) and Light's criteria in seperaing pleural fluid transudates and exudates. Chest 134(4): 1-57. 